\title{
A Diffusion-Augmented Level Set Method with Efficient Two Step Implementation
}

\author{
Naitik D. Kapadia \\ M.tech student, RKDF-IST \\ Bhopal, India
}

\author{
Rinku K. Solanki \\ M.tech student, NRI \\ Bhopal, India
}

\author{
Bhagwan S. Sharma \\ Assistant Professor, RKDF-IST \\ Bhopal, India
}

\begin{abstract}
The level set method was devised by Osher and Sethian [2] in as a simple and versatile method for computing and analyzing the motion of an interface $\Gamma$ in two or three dimensions. $\Gamma$ bounds a region $\Omega$. The goal is to compute and analyze the subsequent motion of $\Gamma$ under a velocity field $v$ [1]. This velocity can depend on position, time, the geometry of the interface and the external physics. The interface is captured for later time as the zero level set of a smooth function $\phi(\mathrm{x}, t)$, i.e., $\Gamma(t)=\{x \mid \phi(\mathrm{x}, t)=0\}$. $\phi$ is positive inside $\Omega$, negative outside $\Omega$ and is zero on $\Gamma(t)$ [1]. This paper presents a reaction-diffusion method used to describe a physicochemical phenomenon that comprises two elements, namely chemical reactions and diffusion for implicit active contours[21][37][39][40], which is completely free of the costly re-initialization procedure in level set evolution (LSE). A diffusion term is introduced into LSE, resulting in a diffusion-augmented level set method with efficient two step implementation. First we iteratively solve the diffusion term and then iteratively solve the level set equation. By solving equation in two steps we can stabilize the level set function without re-initialization. This is also called two step splitting method for image segmentation.
\end{abstract}

\section{General Terms}

Image segmentation, two step splitting method, partial differential equation, active contour

\section{Keywords}

Level set method, image segmentation, diffusion; level set evolution, re-initialization, signed distance function

\section{INTRODUCTION}

Level set methods have seen tremendously applications in many areas over the past decade [13]. This has been made possible by the exibility of the level set formulation in dealing with dynamic evolutions and topological changes of curves and surfaces, and by the mathematical theory and numerical tools developed in studying these methods[15][18][23]. The level set methods (LSM) can be categorized into partial differential equation (PDE) based ones and variational ones.

Level set method was first introduced by Osher and Sethian, and has become a more and more popular theoretical and numerical framework within image processing, fluid mechanics, graphics, computer vision, etc.[2] The level set method is basically used for tracking moving fronts [1-2] by considering the front as the zero level set of an embedded function, called the level set function. In image processing, it is used for propagating curves in $2 \mathrm{D}$ or surfaces in $3 \mathrm{D}$. The applications of the level set method cover most fields in image processing, such as noise removal, image inpainting, image segmentation and reconstruction [13]. In image segmentation, the level set method has some advantages compared to the active contour model. The level set method conquers the difficulties of topological transformations. The level set approach is able to handle complex topological changes automatically [2].To stop evolution of level set function traditionally we are usually find gradient of given image [26], which is less efficient. Later in advancement of level set method, we used different approach to stop evolution of level set function. Chan and Vese, the authors propose a different active contour model that based on the Mumford-Shah functional for segmentation [14], which does not use gradient to stop the evolution. In addition, by using this model and its level set formulation, interior contours are automatically detected, and the initial curve can be anywhere in the image. The liberty of formulation of these level set methods gives us countless possibilities.In recent years, some variational level set [6] formulations have been proposed to regularize the LSF during evolution, and hence the re-initialization procedure can be eliminated. These variational LSMs without reinitialization have many advantages over the traditional methods, including higher efficiency and easier implementation. Chunming Lia proposed new variational formulation for geometric active contours that forces the level set function to be close to a signed distance function [8], and therefore completely eliminates the need of the costly reinitialization procedure. Other problems of Intensity inhomogeneities occur in real-world images and may cause considerable difficulties in image segmentation. Chunming Li a new variational level set formulation in which the regularity of the level set function such that the derived level set evolution has a unique forward-and-backward (FAB) diffusion effect, which is able to maintain a desired shape of the level set function, particularly a signed distance profile near the zero level set. This yields a new type of level set evolution called distance regularized level set evolution (DRLSE) [41].This paper presents a reaction-diffusion method used to describe a physico-chemical phenomenon that comprises two elements, namely chemical reactions and diffusion for implicit active contours, which is completely free of the costly re-initialization procedure in level set evolution (LSE). A diffusion term is introduced into LSE, resulting in a diffusion-augmented level set method with efficient two step implementation, thus re-initialization procedure is completely eliminated from LSE. The rest of the paper is organized as follows. Section 2 introduces the background and related works. Section 3 presents the Reaction-diffusion systems on moving surfaces. Section 4 implements Diffusion based LSM, and analyzes the consistency between theory and implementation. Section 5 presents experimental results and Section 6 concludes the paper. 


\section{BACKGROUND AND RELATED WORKS}

The original idea behind the level set method was a simple one. Given an interface $\Gamma$ in $\mathrm{R}^{\mathrm{n}}$ of codimension one, bounding a open region $\Omega$ [1], we wish to analyze and compute its subsequent motion under a velocity field $\vec{v}$. This velocity can depend on position, time, the geometry of the interface e.g. its normal or its mean curvature and the external physics. The idea, as devised in 1987 by S. Osher and J.A. Sethian [2] is merely to define a smooth function $\phi(x, t)$, that represents the interface as the set where $\phi(\mathrm{x}, \mathrm{t})=0$. Here $\mathrm{x}=\mathrm{x}\left(\mathrm{x}_{1} \ldots \mathrm{x}_{\mathrm{n}}\right) \epsilon$ $\mathrm{R}^{\mathrm{n}}$. The level set function $\phi$ has the following properties

$$
\begin{aligned}
& \phi(\mathrm{x}, \mathrm{t})>0 \text { for } \mathrm{x} \in \Omega \\
& \phi(\mathrm{x}, \mathrm{t})<0 \text { for } \mathrm{x} \notin \bar{\Omega} \\
& \phi(\mathrm{x}, \mathrm{t})=0 \text { for } \mathrm{x} \in \partial \Omega=\Gamma(\mathrm{t})
\end{aligned}
$$

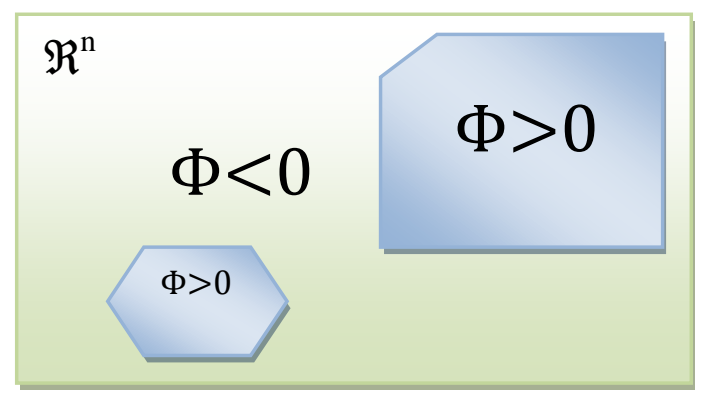

Fig 1: implicit and explicit regions

Hence we can identify the interface by locating the set for which $\Gamma(t)$ for which $\phi$ vanishes. These phenomena can be useful for numerical computation, primarily for topological changes such as breaking and merging [1][2][3]. The motion is analyzed by convecting the $\phi$ values (levels) with the velocity field $\vec{v}$. This elementary equation is

$$
\frac{\mathrm{d} \varphi}{\mathrm{dt}}+\vec{v} \cdot \nabla \varphi=0
$$

Here $\vec{v}$ is the desired velocity on the interface, and is arbitrary elsewhere. Actually, only the normal component of $v$ is needed: $V_{N}=\vec{v} \cdot \nabla \varphi$, so (1) becomes

$$
\frac{\mathrm{d} \varphi}{\mathrm{dt}}+V_{N} \cdot \nabla \varphi=0 .
$$

Since $\mathrm{F}$ is defined as the speed in the outward normal direction, then $\bar{x} \cdot \mathrm{n}=\mathrm{F}$, where $\mathrm{n}=\frac{\Delta \varphi}{|\Delta \varphi|}$. This yields an evolution equation for $\varphi$ :

$$
\frac{\mathrm{d} \varphi}{\mathrm{dt}}+\mathrm{F} \cdot|\nabla \varphi|=0
$$

This equation is defined by the authors "osher and sethian"[2]. Using this equation we can find the topological changes using iteration methods. One disadvantage of this level set method is that level set function (LSF) becomes to flat or too steep near the zero level set, causing numerical errors [15][23]. This problem can be solved by re-initializing the level set function and repeat the same procesedure until find the desired level set.

\subsection{Re-initialization:}

Using re-initialization method we can find topological changes in the image like merging and splitting. a procedure called re-initialization is periodically employed to reshape it to be an SDF [2][8][41]. This method is used extensively with level set method for stable numerical solution. The standard re-initialization method is to solve the following reinitialization equation,

$$
\varphi_{\mathrm{t}}+\operatorname{Sign}\left(\varphi_{0}\right)(1-|\nabla \varphi|)=0
$$

Where $\varphi_{0}$ is the function to be re-initialized, and $\operatorname{sign}(\varphi)$ is the sign function. If $\varphi_{0}$ is not smooth or is too steeper at one side of the interface than the other, the resulting zero level set function may be incorrect from that of the original function. If signed distance function is far away from the level set function then it becomes difficult to re-initialize the level set function to be signed distance function [9]. In practice, the evolving level set function can deviate greatly from its value as signed distance in a small number of iteration steps, especially when the time step is not chosen small enough. Reinitialization has been extensively used as a numerical remedy for maintaining stable curve evolution and having desirable results. The re-initialization process can be quite complicated, expensive and have subtle side effects. Most of the level set methods are fight with their own problems, such as when and how to re-initialize the level set function to a signed distance function.

\subsection{Signed distance function:}

As to get stable and accurate evolution of topological changes we are initializing the level set function as signed distance function. Osher and Sethian proposed to initialize the LSF as $\varphi(\mathrm{x})=1 \pm \operatorname{dist}^{2}(\mathrm{x})$, where $\operatorname{dist}(\cdot)$ is a distance function and " \pm " denotes the signs inside and outside the contour [9]. Later, Mulder et al.initialized the LSF as $\varphi(\mathrm{x})= \pm \operatorname{dist}(\mathrm{x})$, which is an SDF that can result in accurate numerical solutions.reinitialization methods do not directly compute the SDF since the solution of $|\nabla \varphi|=1$ is itself an SDF [28]. In, the following re-initialization equation was proposed

$$
\varphi_{\mathrm{t}}+S\left(\varphi_{0}\right)(|\nabla \varphi|-1)=0
$$

Where $S\left(\varphi_{0}\right) \triangleq \frac{\varphi}{\sqrt{\varphi^{2}+|\nabla \varphi| 2 \cdot(\Delta \mathrm{x}) 2}}, \varphi_{0}$ is the initial LSF and $\Delta x$ is the spatial step. Unfortunately, if the initial LSF $\varphi_{0}$ deviates much from an SDF, Eq. will fail to yield a desirable final SDF.

As re-initialization has many drawbacks such as the expensive computational cost, blocking the emerging of new contours, failures when the LSF deviates much from an SDF, and inconsistency between theory and implementation. Solution to this problem is proposed to regularize the variational LSF to eliminate the re-initialization procedure. $\mathrm{Li}$ proposed the distance regularized level set evolution (DRLSE) method for accurate evolution of LSF [41]. Li et al. proposed a signed distance penalizing energy functional:

$$
\mathrm{P}(\varphi)=\int_{\Omega}(|\nabla \varphi|-1) 2 d x
$$

This equation measures the closeness between an $\operatorname{LSF} \varphi$ and an SDF in the domain $\Omega \subset R^{n}$.Although DRLSE methods have many advantages over re-initialization methods, such as higher efficiency and easier implementation, they still have

\section{Table 1: Comparison of different level set method}

the following drawbacks as limited application to PDE-based LSMs, limited anti-leakage capability for weak boundaries, and sensitivity to noise. By adding the diffusion term into level set method equation and evaluate it in two steps we can get better accuracy and stability in image segmentation and it becomes free from costly re-initialization procedure. 


\begin{tabular}{|c|c|c|}
\hline \multicolumn{3}{|c|}{ Comparison of different level set method } \\
\hline $\begin{array}{l}\text { SR } \\
\text { NO } \\
.\end{array}$ & TITTLE & Proposed Method \\
\hline 1 & $\begin{array}{lr}\text { An Efficient } & \text { Algorithm for } \\
\text { Level Set } & \text { Method } \\
\text { Preserving } & \text { Distance } \\
\text { Function } & \end{array}$ & $\begin{array}{l}\text { Fast algorithm to preserve } \\
\text { distance functions in level } \\
\text { set methods. algorithm is } \\
\text { inspired by recent } \\
\text { efficient } \ell \text { optimization } \\
\text { techniques }\end{array}$ \\
\hline 2 & $\begin{array}{l}\text { Re-initialization Free Level } \\
\text { Set Evolution via Reaction } \\
\text { Diffusion }\end{array}$ & $\begin{array}{l}\text { This paper presents a novel } \\
\text { reaction diffusion (RD) } \\
\text { method for implicit active } \\
\text { contours, which is } \\
\text { completely free of the } \\
\text { costly re-initialization } \\
\text { procedure in level set } \\
\text { evolution (LSE). A } \\
\text { diffusion term is } \\
\text { introduced into LSE, } \\
\text { resulting in a RD-LSE } \\
\text { equation. }\end{array}$ \\
\hline 3 & $\begin{array}{l}\text { Distance Regularized Level } \\
\text { Set Evolution and Its } \\
\text { Application to Image } \\
\text { Segmentation }\end{array}$ & $\begin{array}{l}\text { The DRLSE defined with a } \\
\text { potential function such that } \\
\text { the derived LSE has a } \\
\text { unique forward-and- } \\
\text { backward (FAB) diffusion } \\
\text { effect, which is able to } \\
\text { maintain a desired shape of } \\
\text { the LSF, particularly a } \\
\text { signed distance profile } \\
\text { near the zero level set. }\end{array}$ \\
\hline 4 & $\begin{array}{l}\text { A Level Set Method for } \\
\text { Image Segmentation in the } \\
\text { Presence of Intensity } \\
\text { Inhomogeneities With } \\
\text { Application to MRI }\end{array}$ & $\begin{array}{l}\text { This paper proposes a } \\
\text { novel region-based method } \\
\text { for image segmentation, } \\
\text { which is able to deal with } \\
\text { intensity in homogeneities } \\
\text { in the segmentation. }\end{array}$ \\
\hline 5 & $\begin{array}{l}\text { Modified Gradient Search } \\
\text { for Level Set Based Image } \\
\text { Segmentation }\end{array}$ & $\begin{array}{l}\text { Active contours, gradient } \\
\text { methods: (1) resilient } \\
\text { propagation (Rprop) (2) } \\
\text { using a momentum term } \\
\text { image segmentation, level } \\
\text { set method, machine } \\
\text { learning, optimization, } \\
\text { variational problems. }\end{array}$ \\
\hline
\end{tabular}

\section{REACTION-DIFFUSION SYSTEM ON MOVING SURFACES}

The term reaction-diffusion system is basically related to physico-chemical phenomenon that comprises two elements, namely chemical reactions and diffusion [16][21]. A chemical reaction system consists of $\mathrm{N}$ species $\left(\mathrm{x}_{1} \ldots \mathrm{x}_{\mathrm{n}}\right)$ (e.g. molecules) together with $\mathrm{M}$ reaction channels, $\left(\mathrm{r}_{1} \ldots \mathrm{r}_{\mathrm{m}}\right.$. . Each reaction channel defines the stoichiometry of a reaction

$$
\mathrm{r}_{\mathrm{m}}: \sum_{i} \propto_{\mathrm{im}} \mathrm{X}_{\mathrm{i}} \stackrel{k m}{\rightarrow} \sum_{j} \beta_{\mathrm{jm}} \mathrm{X}_{\mathrm{j}}
$$

This describes the idea that whenever the species $\mathrm{i}$ come together with molar concentrations $\propto_{i}$, they are interconverted
International Journal of Computer Applications (0975 - 8887)

Volume 70-No.23, May 2013

to the species $\mathrm{j}$ with molar concentrations $\beta_{\mathrm{j}}$ with a specific reaction rate $\mathrm{k}_{\mathrm{m}}$. Using the law of mass action , one can derive a system

$$
\frac{d c}{d t}=\sum_{1}^{M} \mathrm{k}_{\mathrm{m}}\left(\beta_{\mathrm{im}}-\propto_{\mathrm{im}}\right)
$$

or in matrix notation

$$
\frac{d c}{d t}=\mathrm{M}_{\mathrm{S}} \cdot \mathrm{r}(\mathrm{t})
$$

Where $\mathrm{M}_{\mathrm{S}}$ is the stoichiometric matrix and $\mathrm{r}$ the rate vector describing the speed of each reaction.

The second phenomenon, diffusion, refers to the process of thermal motion of molecules [38][39][40]. It is the process by which for example warm and cold water intermingles until the water has a uniform temperature (at thermal equilibrium) or by which a fragrant smell spreads in a room. The general macroscopic diffusion equation for species $\mathrm{i}=1,2, \ldots . \mathrm{N}$ is

$$
\frac{d c}{d t}=\nabla \cdot\left(\mathrm{D}_{\mathrm{i}} \nabla \mathrm{c}_{\mathrm{i}}\right)
$$

where Di denotes the diffusion tensor of species i, a matrix that defines how well the molecule i diffuses into the different spatial directions.

Combining equations and, we get the so called reactiondiffusion equation. The equations for a reaction-diffusion system on a surface $\Gamma \subset \Omega \subseteq \mathrm{R}^{3}$ are then

$$
\frac{d c}{d t}=\mathrm{R}_{\mathrm{i}}(\mathrm{C})+\mathrm{D}_{\mathrm{i}} \Delta_{\Gamma} \mathrm{c}_{\mathrm{i}} \quad \mathrm{i}=1,2 \ldots \ldots \mathrm{N} ;
$$

Since the zero level is used to represent the object contour, we only need to consider the zero level set of the LSF. We can use a function with different phase fields as the LSF. Motivated by the phase transition theory, by adding a diffusion term into the conventional LSE equation. Such an introduction of diffusion to LSE will make LSE stable without re-initialization. By adding a diffusion term " $\varepsilon \Delta \varphi$ " into the LSE equation, we have the following RD equation for LSM:

$$
\varphi_{\mathrm{t}}=\varepsilon \Delta \varphi-\frac{1}{\varepsilon} \mathrm{L}(\varphi), \mathrm{x} \in \Omega \subset \mathrm{R}^{\mathrm{n}}
$$

\section{TWO STEP DIFFUSION BASED LSM}

A Two step algorithm to implement Diffusion has been proposed in to generate the curvature-dependent motion. In the reaction function is first forced to generate a binary function with values 0 and 1 , and then the diffusion function is applied to the binary function to generate curvaturedependent motion. Different from, where the diffusion function is used to generate curvature-dependent motion, in our proposed LSM, the LSE is driven by the reaction function, i.e., the LSE equation. Therefore, we propose to use the diffusion function to regularize the LSF generated by the reaction function. To this end, we propose the following two step method to solve the equation.

Step 1: Solve the reaction term till some time $T_{r}$ to obtain the intermediate solution, denoted by $\varphi n+1 / 2=\varphi^{\mathrm{n}}$;

Step 2: Solve the diffusion term $\varphi \mathrm{t}=\varepsilon \Delta \varphi, \varphi(\mathrm{x}, \mathrm{t}=0)=\varphi n+1 / 2$ till some time $T_{d}$, and then the final level set is $\varphi n+1$ $=\varphi(x, T d)$

\subsection{PROPOSED ALGORITHM:}

1. Read image

2. Apply Gaussian kernel for smoothing 
3. Implementation of Dirac function $\delta(x)$

4. Selection of Time Step value

5. Implementation of penalizing energy term $\mathrm{P}(\backslash \mathrm{phi})$ For implementation of penalizing) energy term $\mathrm{P}(\backslash \mathrm{phi})$ Set following parameter

- $\quad \mathrm{u} 0$ : level set function to be updated

- $\quad$ : edge indicator function

- lambda: coefficient of the weighted length term L(\phi)

- mu: coefficient of the internal (penalizing) energy term $\mathrm{P}($ (phi)

- alf: coefficient of the weighted area term $\mathrm{A}(\backslash \mathrm{phi})$, choose smaller alf

- epsilon: the papramater in the definition of smooth Dirac function, default value 1.5

- delt: time step of iteration, see the paper for the selection of time step and mu

- numIter: number of iterations.

6. Define initial level set function

The initial LSF

$$
\Phi_{0}\left(\mathrm{x}_{1}, \mathrm{x}_{2}\right)=\sqrt{(\mathrm{x} 1-50) 2+(x 2-50) 2}-30
$$

7. Start level set evolution

8. Optimization of results (final contour detection.)

\section{EXPERIMENTAL RESULTS}
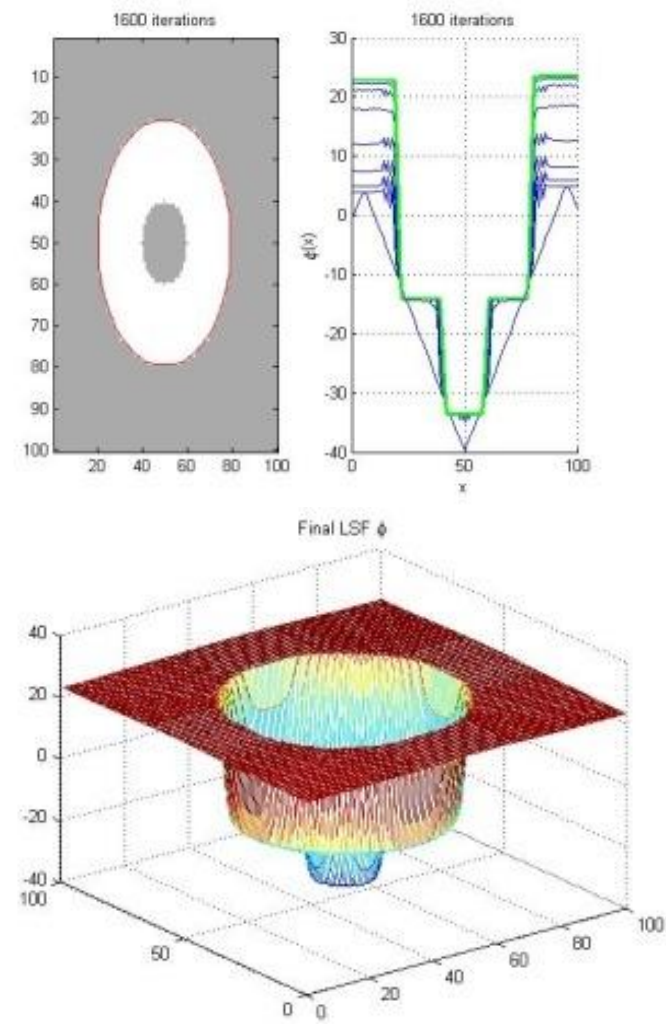

Fig 2: (a) Testing image. red circle represents the initial contour and Middle slices of level set function during LSE. (b) The final LSFs .The initial LSF is $\Phi_{0}\left(x_{1}, x_{2}\right)=$
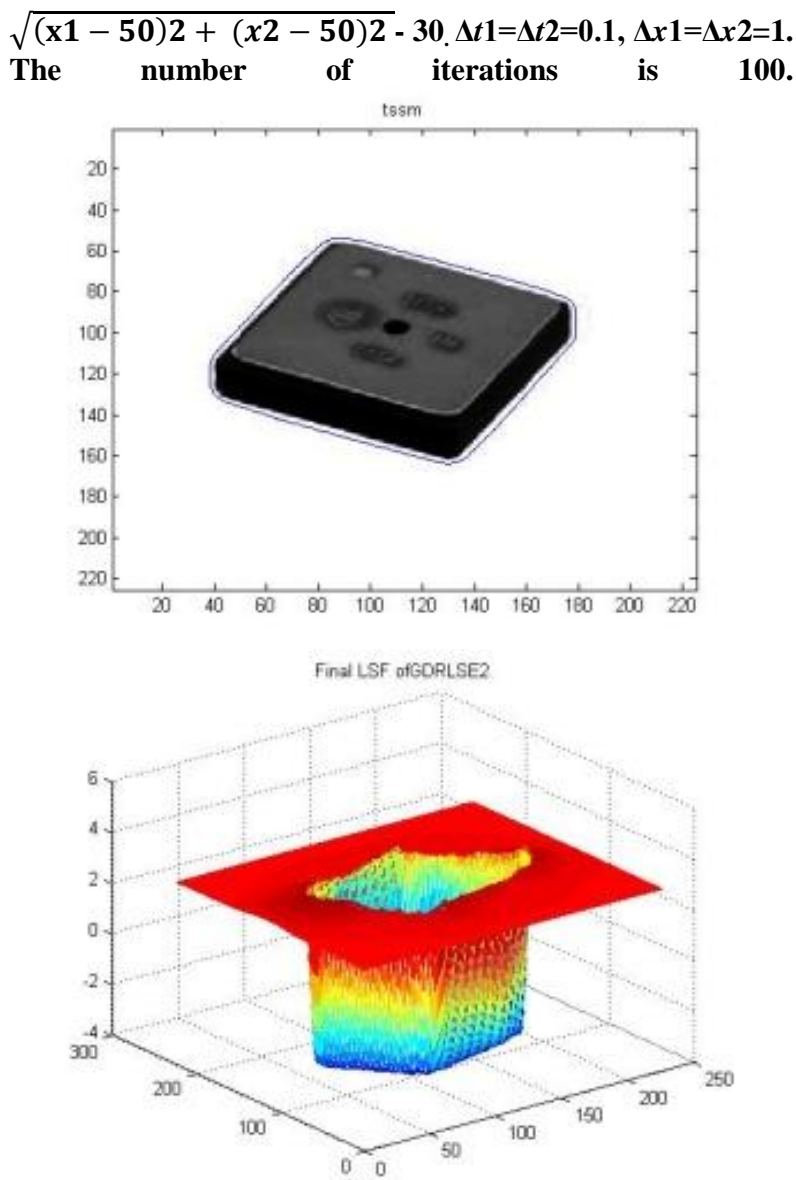

Fig 3: (a) Testing image. (b) The final LSFs. image size = $225 \times 225$, type: $\operatorname{bmp}, \operatorname{Time} \operatorname{step}(\Delta T)=1, \operatorname{Mu}(\mu)=0.2 / \Delta T$, Lambda $(\gamma)=5$, Alpha $(\propto)=-0.3$, Epsilon $(\epsilon)=1.5$, Sigma $(\sigma)=0.8$, iteration $=1500$, segment time $=2.03 .04$ second
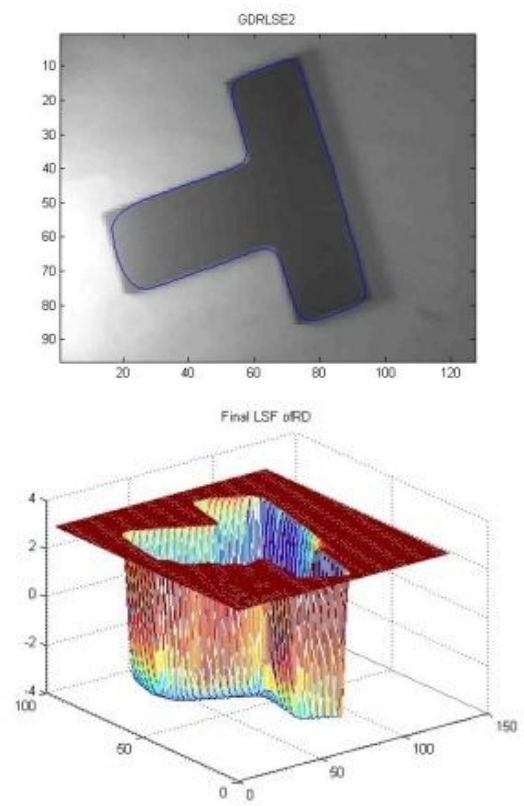

Fig 4: (a) Testing image. (b) The final LSFs. Image size $=$ $127 \times$ 96, type: bmp, Time step $(\Delta T)=1, M u(\mu)=0.2 / \Delta T$, Lamda $(\gamma)=5$, Alpha $(\propto)=-0.3$, Epsilon $(\epsilon)=1.5$, Sigma $(\sigma)=0.8$, iteration $=1500$, segment time $=1.36 .08$ second 

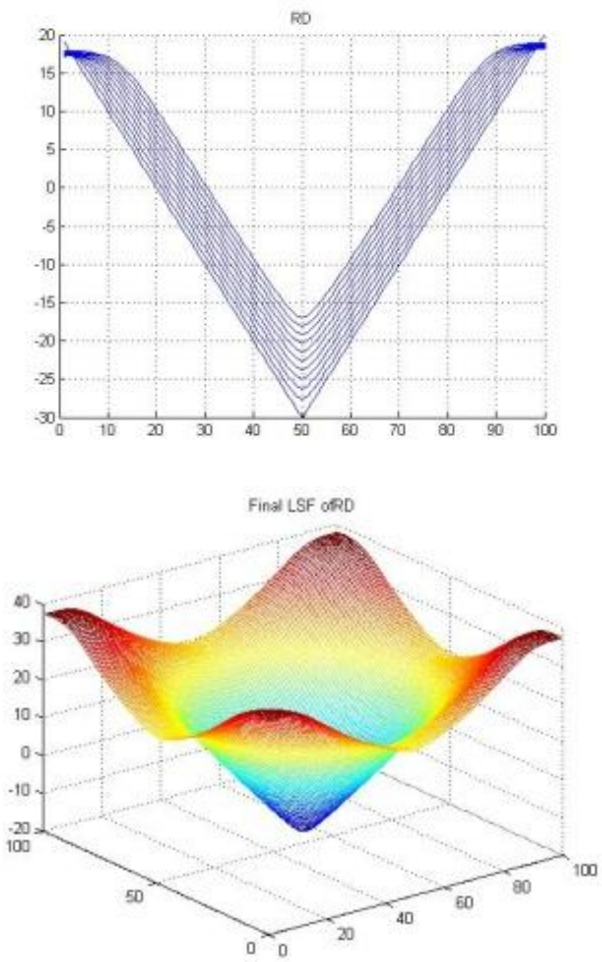

Fig 5: Two step diffusion based LSM method (a) middle slices of the LSFs in iterations (b) the final LSFs. We set $\Delta t 1=\Delta t 2=0.1$.
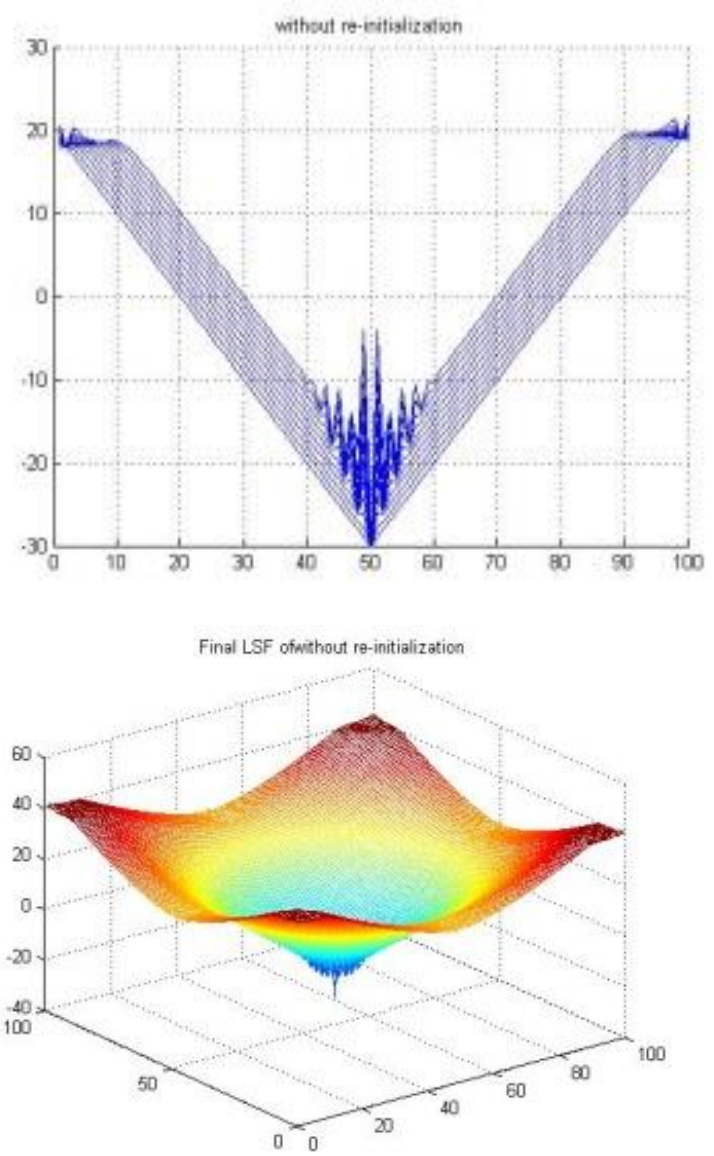

Fig 6: DRLSE method (a) middle slices of the LSFs in iterations (b) the final LSFs. We set $\Delta t 1=\Delta t 2=0.1$

\begin{tabular}{|l|l|l|l|l|l|l|l|}
\hline & $\Delta T$ & $\begin{array}{l}\mathrm{Mu} \\
(\mu)\end{array}$ & $(\gamma)$ & $(\propto)$ & $(\in)$ & $(\sigma)$ & time \\
\hline $\begin{array}{l}\text { DIFFU- } \\
\text { LSM }\end{array}$ & 0.1 & $0.2 / \Delta T$ & 5 & -0.3 & 1.5 & 0.8 & 4.8 \\
\hline DRLSE & 0.1 & $0.2 / \Delta T$ & 5 & -0.3 & 1.5 & 0.8 & 6.2 \\
\hline
\end{tabular}

Table 2: Comparison of experimental results of Diffusion based LSM and DRLSE method.

\section{CONCLUSION}

The Two step diffusion based LSM is general, which can be applied to the PDE-based level set methods and variational ones. Second, method has much better performance on weak boundary anti-leakage. Third, the implementation of the method is very simple and it does not need the upwind scheme at all. Fourth, this method is robust to noise. The experiments on synthetic and real images demonstrated the promising performance of our approach.

\section{ACKNOWLEDGMENTS}

Thanking to Ass. Prof. Bhagwan S. Sharma, for his valuable knowledge and support and guiding us to the right path.

\section{REFERENCES}

[1] M. Kass, A. Witkin, and D.Terzopoulos, "Snakes: Active contour models," Int. J. Comput. Vis., vol.1, pp. 321331,1987 .

[2] S. Osher and J. Sethian, "Fronts propagating with curvature dependent speed: Algorithms based on Hamilton-Jacobi formulations," J. Comp. Phys., vol. 79, pp. 12-49, 1988.

[3] V. Caselles, F. Catte, T. Coll, and F. Dibos, "A geometric model for active contours in image processing," Numer. Math., vol. 66, pp. 1-31, 1993

[4] R. Malladi, J. Sethian, and B. Vemuri, "Shape Modeling with Front Propagation: A Level Set Approach," IEEE Trans. Pattern Analysis and Machine Intelligence, vol. 27, no. 5, pp. 793-800, 1995.

[5] V. Caselles, R. Kimmel, and G. Sapiro, "Geodesic Active Contours," Int. J. Comput. Vis., vol. 22, no. 1 pp. 61-79,1997.

[6] H. Zhao, T. Chan, B. Merriman, and S. Osher, "A Variational Level Set Approach to Multiphase Motion," J. Comp. Phys., vol. 127, pp. 179-195, 1996.

[7] D. Peng, B. Merriman, S. Osher, H. Zhao, and M. Kang, “A PDE-Based Fast Local Level Set Method," J. Comp. Phys., vol. 155, pp. 410-438, 1999.

[8] C. Li, C. Xu, C. Gui, and M. D. Fox, "Level set evolution without re-initialization: A new variational formulation,"Proc. IEEE Conf. Computer Vision and Pattern Recognition, vol. 1, pp. 430-436, 2005.

[9] S. Osher and R. Fedkiw, Level Set Methods and Dynamic Implicit Surfaces, Springer-Verlag, New York, 2002. 
[10] B. Merriman, J. Bence, and S. Osher, "Motion of Multiple Junctions: A Level Set Approach," J. Comp. Phys., vol. 112, pp. 334-363, 1994

[11] K. Zhang, L. Zhang, H. Song and W. Zhou, "Active contours with selective local or global segmentation: a new formulation and level set method," Image and Vision Computing, vol. 28, issue 4, pp. 668-676, April 2010.

[12] M. Sussman, P. Smereka, S. Osher, "A Level Set Approach for Computing Solutions to Incompressible Two-Phase Flow," J. Comp. Phys., vol. 114, pp. 146159, 1994.

[13] R. Tsai, and S. Osher, "Level Set Methods and Their Applications in Image Science," COMM.MATH.SCI., vol.1, no. 4, pp. 623-656, 2003.

[14] T. Chan and L. Vese, "Active contours without edges," IEEE Trans.Image Process, vol. 10, no. 2, pp. 266-277, Feb. 2001.

[15] G. Aubert and P. Kornprobst, Mathematical problems in image processing, New York: Springer-Verlag, 2000

[16] J. Rubinstein, P. Sternberg, and J. Keller, "Fast reaction, slow diffusion, and curve shortening," SIAM J.APPL.MATH, Vol. 49, No. 1, pp. 116-133, Feb. 1989.

[17] J. Xu, H. Zhao, "An Eulerian Formulation for Solving Partial Differential Equations Along a Moving Interface,” J. Sci. Comp., vol. 19, pp. 573-594, 2003.

[18] J. Strikwerda, Finite difference schemes and partial differential equations, Wadsworth \& Brooks/Cole Advanced Books \& Software, Pacific grove, California, 1989.

[19] S. Allen and J. Cahn, "A Microscopic Theory for Antiphase Boundary Motion and Its Application to Antiphase Domain Coarsening," Acta Metallurgica., vol. 27, pp. 1085-1095, 1979.

[20] S. Baldo, "Minimal Interface Criterion for Phase Transitions in Mixtures of Cahn-Hilliard Fluids," Annals Inst. Henri Poincare., vol. 7, pp. 67-90, 1990.

[21] G. Barles, L. Bronsard, and P. Souganidis, "Front Propagation for Reaction-Diffusion Equations of Bistable Type,"Annals Inst. Henri Poincare., vol. 9, pp. 479-496, 1992.

[22] I. Fonseca and L. Tartar, "The Gradient Theory of Phase Transitions for Systems with Two Potential Wells," Proc. Royal Soc. Edinburgh., vol. 111A, no. 11, pp. 89-102, 1989.

[23] http://www.engr.uconn.edu/ cmli/

[24] C. Li, C. Kao, J. Gore, and Z. Ding, "Implicit Active Contours Driven by Local Binary Fitting Energy," Proc. IEEE Conf. Computer Vision and Pattern Recognition, pp. 1-7, 2007.

[25] L. Modica, "The Gradient Theory of Phase Transitions and the Minimal Interface Criterion," Arch. Rational Mech. Anal., vol. 98, pp. 123-142, 1987.
[26] X. Xie, "Active Contouring Based on Gradient Vector Interaction and Constrained Level Set Diffusion," IEEE Trans. Image Processing, vol. 19, no. 1, pp. 154-164, Jan. 2010.

[27] D. Chopp, "Computing Minimal Surface via Level Set Curvature Flow," J.Comput.Phys., vol. 106, pp. 77-91, 1993.

[28] W. Mulder, S. Osher and J. Sethian, "Computing Interface Motion in Compressible Gas Dynamics," J.Compt. Phys., vol. 100, pp. 209-228, 1992.

[29] G. Russo and P. Smereka, "A Remark on Computing Distance Functions," J.Comput. Phys., vol. 163, pp. 5167, 2000.

[30] M. Sussman and E. Fatemi, "An Efficient InterfacePreserving Level Set Redistancing Algorithm and Its Application to Interfacial Incompressible Fluid Flow," SIAM J.Sci. Comput., vol. 20, pp. 1165-1191, 1999.

[31] J. Gomes and O. Faugeras, "Reconciling distance functions and Level Sets," J.Visiual Communic. And Imag. Representation, vol. 11, pp. 209-223, 2000.

[32] L. Vese and T. Chan, "A multiphase level set framework for image segmentation using the Mumford-Shah model,” Int. J. Comput. Vis., vol. 50, pp. 271-293, 2002.

[33] S. Ruuth, "A diffusion-generated approach to multiphase motion," J.Comput. Phys., vol. 145, pp. 166-192, 1998.

[34] ]S. Ruuth, B. Merriman, "Convolution generated motion and generalized huygens's principles for interface motion,"

[35] B. Merriman and S. Ruuth, "Diffusion generated motion of curves on surfaces," J.Comput. Phys., vol. 225, pp.2267-2282, 2007.

[36] S. Ruuth, "Efficient algorithm for diffusion-generated motion by mean curvature," J.Comput. Phys., vol. 144, pp.603-625, 1998.

[37] S. Zhu and D. Mumford, "Prior Learning and Gibbs Reaction-Diffusion," IEEE Trans. Pattern Analysis and Machine Intelligence, vol. 19, no. 11, pp. 1236-1250, 1997.

[38] G. Turk, "Generating Textures on Arbitrary Surfaces Using Reaction-Diffusion,” Computer Graphics, vol. 25, no. $4,1991$.

[39] A. Witkin, and M. Kass, "Reaction-diffusion textures," ACM SIGGRAPH, 1991.

[40] A. Sanderson, M. Kirby, C. Johnson, and L. Yang, "Advanced Reaction-Diffusion Models for Texture Synthesis," Journal of Graphics Tools, vol. 11, no. 3, pp. 47-71, 2006.

[41] C. Li, C. Xu, C. Gui, and M. D. Fox, "Distance Regularized Level Set Evolution and Its Application to Image Segmentation," IEEE Trans. Image Processing, vol. 19 , no. 12, pp. 154-164, Dec. 2010. 